\title{
Inspiratory flow limitation during sleep in pre-eclampsia: comparison with normal pregnant and nonpregnant women
}

\author{
G. Connolly*, A.R.A. Razak*, A. Hayanga*, A. Russell ${ }^{\#}$, P. McKenna*, W.T. McNicholas
}

\begin{abstract}
Inspiratory flow limitation during sleep in pre-eclampsia: comparison with normal pregnant and nonpregnant women. G. Connolly, A.R.A. Razak, A. Hayanga, A. Russell, P. McKenna, W.T. McNicholas. (C) ERS Journals Ltd 2001.

ABSTRACT: Self-reported snoring is common in pregnancy, particularly in females with pre-eclampsia. The prevalence of inspiratory flow limitation during sleep in preeclamptic females was objectively assessed and compared with normal pregnant and nonpregnant females.

Fifteen females with pre-eclampsia were compared to 15 females from each of the three trimesters of pregnancy, as well as to $\mathbf{1 5}$ matched nonpregnant control females (total study population, 75 subjects). All subjects had overnight monitoring of respiration, oxygen saturation, and blood pressure (BP).

No group had evidence of a clinically significant sleep apnoea syndrome, but patients with pre-eclampsia spent substantially more time $(31 \pm 8.4 \%$ of sleep period time, mean \pm SD) with evidence of inspiratory flow limitation compared to $15.5 \pm \mathbf{2 . 3} \%$ in third trimester subjects and $<5 \%$ in the other three groups $(\mathrm{p}=\mathbf{0 . 0 0 1})$. In the majority of preeclamptics, the pattern of flow limitation was of prolonged episodes lasting several minutes without associated oxygen desaturation. As expected, systolic and diastolic BPs were significantly higher in the pre-eclamptic group $(\mathbf{p}<0.001)$, but all groups showed a significant fall $(\mathbf{p} \leqslant \mathbf{0 . 0 5})$ in $\mathrm{BP}$ during sleep.

Inspiratory flow limitation is common during sleep in patients with pre-eclampsia, which may have implications for the pathophysiology and treatment of this disorder.
\end{abstract} Eur Respir J 2001; 18: 672-676.

*Depts of Obstetrics and Gynaecology, Rotunda Hospital, and "The Respiratory Sleep Disorders Unit, St. Vincent's University Hospital, Dublin, Ireland.

Correspondence: W.T. McNicholas, Dept of Respiratory Medicine, St. Vincent's University Hospital, Elm Park, Dublin 4, Ireland.

Fax: 35312697949

Keywords: Hypertension inspiratory flow limitation pre-eclampsia pregnancy sleep apnoea

Received: June 162000 Accepted after revision April 42001
Obstructive sleep apnoea syndrome (OSAS) affects $\leqslant 4 \%$ of middle-aged and older males, but epidemiological data indicate that the condition is much less common in females of childbearing age $[1,2]$. However, snoring has been reported to be particularly common in pregnant females [3, 4], and particularly so in those with pre-eclampsia [4]. These findings raise the possibility that disordered breathing during sleep may be more common in pregnancy, and particularly among females with pre-eclampsia. Since OSAS has been identified as an independent risk factor for the development of hypertension [5,6], it is possible that occult disordered breathing during sleep may be a risk factor to the development of pregnancy-related hypertension, including pre-eclampsia.

Pre-eclampsia is a common complication of pregnancy [7], but its aetiology and pathophysiology are poorly understood $[8,9]$. Recent evidence has emerged that inspiratory flow limitation is common in patients with pre-eclampsia and that short-term relief of flow limitation by nasal continuous positive airway pressure (nCPAP) is associated with a reduction in nocturnal blood pressure (BP) levels in these patients [10]. However, no previous report has compared the prevalence of objective sleep-related breathing abnormalities between females with pre-eclampsia and normal, pregnant females. The authors performed such a study by prospectively studying breathing and oxygenation during sleep in a group of pregnant females, and comparing those with normal pregnancies to those suffering from pre-eclampsia.

\section{Methods}

A case control prospective study involving 75 subjects was performed. Sixty pregnant females attending a University Maternity Hospital were enrolled, 15 normal females from each trimester of pregnancy and 15 females with pre-eclampsia. Preeclampsia was diagnosed according to standard clinical criteria, namely a resting BP $>140 / 90 \mathrm{mmHg}$ together with a 24-h urinary protein level $>0.3 \mathrm{~g}$. Furthermore, each patient with pre-eclampsia had shown a rise in diastolic pressure $>15 \mathrm{mmHg}$ or a rise in systolic pressure $>30 \mathrm{mmHg}$ compared to their initial presentation in the first trimester. None of the pre-eclamptic patients had pre-existing hypertension. Fifteen normal, nonpregnant females of similar age and body mass index (BMI) were also enrolled to serve as control subjects. All subjects selected fulfilled the following inclusion criteria: 1) aged 19-39 yrs; 2) of normal or corresponding age for the stage of pregnancy; and 3) no known history of sleep apnoea or other sleep disorder prior to pregnancy. Case subjects were approached via selective sequencing 
from the antenatal clinic and ward list. Control subjects were obtained by selective sequencing from the student population of the Medical School.

All subjects underwent overnight limited sleep studies between 22:00 and 08:00 $\mathrm{h}$ in a specially designated single room of the hospital. This environment ensured a similar environment for all subjects, and also minimized sleep disturbance due to extraneous noise. Sleep studies were performed using a commercial device (Autoset Portable II Plus ${ }^{\mathrm{TM}}$, ResMed, Sydney, Australia), which provides continuous monitoring of respiration, respiratory effort, body position and arterial oxygen saturation $\left(\mathrm{Sa}_{\mathrm{a}} \mathrm{O}_{2}\right)$ by means of nasal cannulae and abdominal belts attached to the subject, in addition to a pulse oximeter. The Autoset device enables the differentiation of central and obstructive apnoea and hypopnoeas, and also gives an index of inspiratory flow limitation, referred to as the flattening index [11]. This index gives a measure of inspiratory flow limitation that is derived from the relationship of inspiratory flow to inspiratory duration. Previous reports have shown that a flattening index $<0.15$ indicates significant flow limitation, which can be relieved by nCPAP [11], and this was the threshold used in the present report to define significant inspiratory flow limitation. The Autoset device has also been shown to compare well with full polysomnography in the identification of apnoea and hypopnoea frequency during sleep [12, 13]. The Autoset was calibrated by a computer set in the diagnostic mode and provided continuous breath-by-breath recordings of nasal ventilation, abdominal respiratory effort, $\mathrm{Sa}, \mathrm{O}_{2}$, heart rate, snoring level, flattening index, apnoeas, hypopnoeas, respiratory irregularities, and body position. The data were downloaded onto a personal computer, and the differentiation of central and obstructive apnoeas was made by comparing each episode of apnoea with abdominal respiratory effort. The persistence of respiratory effort in the presence of apnoea or hypopnoea indicated an obstructive event. With this device, hypopnoea is defined as a decrease in ventilation $>50 \%$ for $\geqslant 10 \mathrm{~s}$ and apnoea as a decrease in ventilation $>75 \%[12,13]$. Records were reviewed by an experienced sleep technician to improve accuracy of interpretation. Periods of technical artefact were excluded from the analysis.
Each subject also had overnight BP monitoring on the same night, using an ambulatory device that provided hourly BP readings via an automatically inflated arm cuff (Spacelabs Inc., Redmond, WA, USA). BP recordings were taken in bed for $1 \mathrm{~h}$ presleep (before the bedroom light was switched off), during the sleep period time and for $2 \mathrm{~h}$ in bed after sleep. The data were downloaded onto a computer. The choice of hourly readings was a compromise between the need to gain adequate information on BP changes during sleep and the desire to minimize sleep disturbance from the inflation of the arm cuff [14]. Awake BP levels in pre-eclamptic patients were calculated as the mean of 4-hourly resting awake BP recordings taken during the daytime while in hospital.

Statistical analysis was performed using analysis of variance (ANOVA) and paired t-testing as appropriate. The Rotunda Hospital Ethics Committee approved the study protocol and all subjects gave written informed consent. $p$-Values $<0.05$ were regarded as statistically significant and data are presented as mean \pm SD.

\section{Results}

All subjects completed the protocol, and anthropometric and other clinical details of the study population are given in table 1 . Subjects in each group were of similar age, and control subjects were of a similar BMI as subjects in the first trimester. As would be expected, patients in the third trimester and those with pre-eclampsia were heavier than other groups. Preeclamptic patients were of similar gestational age (mean 255 days) as normal third trimester subjects (mean 244 days).

Apnoeas and hypopnoeas were infrequent in all categories during sleep, although central apnoeas were slightly more frequent in the control and first trimester groups. Details of inspiratory flow limitation, as determined by the flattening index, are also given in table 1, which demonstrates that patients with preeclampsia spent almost one-third of the sleep period time with evidence of inspiratory flow limitation, in contrast to $15 \%$ in normal third trimester females, and $<5 \%$ in the other three groups ( $\mathrm{p}=0.001$ by ANOVA).

Table 1. - Anthropometric and sleep-related details of the five study groups

\begin{tabular}{|c|c|c|c|c|c|c|}
\hline \multirow[t]{2}{*}{ Variable } & \multicolumn{5}{|c|}{ Study group } & \multirow[t]{2}{*}{ p-value } \\
\hline & Control & $\mathrm{T} 1$ & $\mathrm{~T} 2$ & T3 & PET & \\
\hline Age yrs & $24.5 \pm 1.2$ & $26.0 \pm 2.4$ & $25.5 \pm 2.1$ & $26.4 \pm 3.2$ & $28.0 \pm 2.3$ & 0.37 \\
\hline BMI & $21.8 \pm 1.4$ & $22.1 \pm 1.3$ & $25.0 \pm 1.7$ & $28.5 \pm 1.8$ & $31.0 \pm 1.3$ & $<0.001$ \\
\hline Total apnoeas & $1.3 \pm 0.2$ & $2.3 \pm 0.3$ & $1.3 \pm 0.3$ & $1.4 \pm 0.4$ & $2.6 \pm 0.5$ & 0.14 \\
\hline Obstructive apnoeas & $0.05 \pm 0.02$ & $0.8 \pm 0.3$ & $1.1 \pm 0.3$ & $1.2 \pm 0.5$ & $1.7 \pm 0.5$ & 0.29 \\
\hline Central apnoeas & $1.3 \pm 0.3$ & $1.5 \pm 0.5$ & $0.2 \pm 0.1$ & $0.2 \pm 0.1$ & $0.9 \pm 0.3$ & 0.015 \\
\hline Hypopnoeas & $0.2 \pm 0.1$ & $0.1 \pm 0.1$ & 0 & 0 & $0.3 \pm 0.2$ & 0.5 \\
\hline Flattening index $\% \mathrm{SPT}$ & $1.6 \pm 0.3$ & $3.7 \pm 0.4$ & $4.6 \pm 0.8$ & $15.5 \pm 2.3$ & $31.0 \pm 8.4$ & 0.001 \\
\hline${\mathrm{Sa}, \mathrm{O}_{2}}_{2}<95 \% \% \mathrm{SPT}$ & $5.3 \pm 1.2$ & $5.6 \pm 2.1$ & $5.4 \pm 0.7$ & $16.2 \pm 2.9$ & $16.7 \pm 3.8$ & 0.06 \\
\hline
\end{tabular}

Data are presented as mean \pm SD unless otherwise stated. BMI: body mass index; \%SPT: per cent sleep period time; T1: first trimester; T2: second trimester; T3: third trimester; PET: pre-eclamptic toxaemia; $\mathrm{Sa}_{\mathrm{a}} \mathrm{O}_{2}$ : arterial oxygen saturation. $\mathrm{p}$-Values refer to analysis of variance (ANOVA) comparing the five subgroups. 


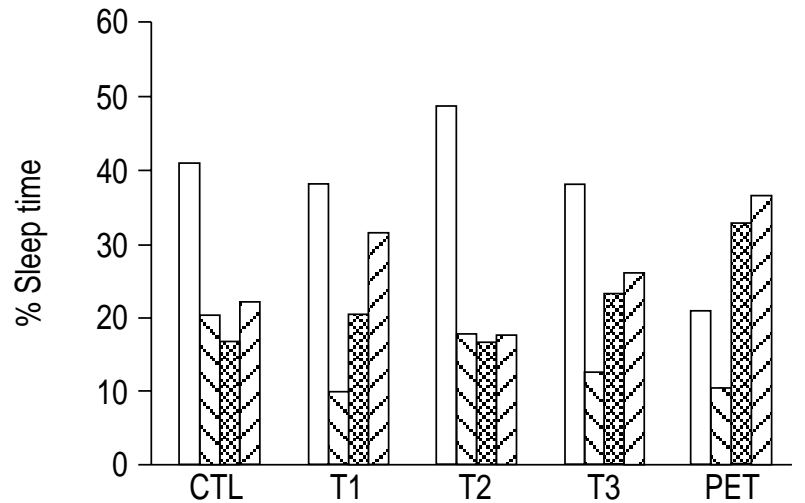

Fig. 1.-Proportion of sleep period time spent in different body positions ( $\square$ : back; $\nabla$ : front; 泶: left; $\square$ : right). CTL: control patients; T1: subjects in first trimester; T2: subjects in second trimester; T3: subjects in third trimester; PET: pre-eclamptic toxaemia patients.

In addition, patients in the third trimester and those with pre-eclampsia tended to spend more time with $\mathrm{Sa}, \mathrm{O}_{2}<95 \%$ than other groups (table 1 ). The greater proportion of time spent with evidence of inspiratory flow limitation in the pre-eclamptic group was not a reflection of body position since this group spent less time in the supine position than subjects without preeclampsia (fig. 1).

Details of BP changes are given in table 2. As would be expected from the patient populations, both systolic and diastolic pressures were significantly higher in the pre-eclamptic group $(\mathrm{p}<0.001$, by ANOVA). Diastolic BP fell significantly $(p \leqslant 0.05)$ in all groups during sleep, compared to when awake, and systolic pressure fell during sleep in all pregnant groups, but not in controls. The hourly changes in systolic and diastolic pressures for the pre-eclamptic group are given in figure 2. Data for the patients with pre-eclampsia are given in table 3 . These data show that the degree of inspiratory flow limitation did not correlate with the severity of hypertension, age, gestational age or BMI.

\section{Discussion}

The present data indicate that inspiratory flow limitation during sleep is common among females with pre-eclampsia in contrast to females with normal pregnancies. These findings suggest a possible relationship between disordered breathing during sleep and pre-eclampsia, but do not allow the authors to determine whether this finding is a causative factor or simply a consequence of the development of preeclampsia. The generalized oedema that is associated with pre-eclampsia [7, 8] could conceivably cause narrowing of the upper airway and thus, predispose to inspiratory flow limitation, thereby favouring a secondary association. However, previous reports have indicated that the development of snoring in

Table 2. - Blood pressure (BP) in the five study groups

\begin{tabular}{lrrrrrr}
\hline Variable & \multicolumn{3}{c}{ Study group } & p-value \\
\cline { 2 - 5 } & Control & T1 & T2 & T3 & PET \\
\hline Systolic BP* & $118 \pm 8.6$ & $122 \pm 6.2$ & $118 \pm 5.2$ & $120 \pm 7.2$ & $152 \pm 5.8$ & $<0.001$ \\
Systolic BP & $116 \pm 8.2$ & $118 \pm 9.1$ & $116 \pm 6.2$ & $116 \pm 10.2$ & $134 \pm 5.7$ & $<0.001$ \\
Diastolic BP* & $77 \pm 6.6$ & $74 \pm 6.7$ & $71 \pm 7.2$ & $77 \pm 5.2$ & $96 \pm 3.2$ & $<0.001$ \\
Diastolic BP & $68 \pm 8.4$ & $67 \pm 8.3$ & $62 \pm 5.2$ & $64 \pm 5.8$ & $76 \pm 6.1$ & $<0.001$ \\
\hline
\end{tabular}

Data are presented as mean \pm SD unless otherwise stated. T1: first trimester; T2: second trimester; T3: third trimester; PET: pre-eclamptic toxaemia. *: awake. ${ }^{\#}$ : asleep. p-Values refer to analysis of variance (ANOVA) comparing the five subgroups.

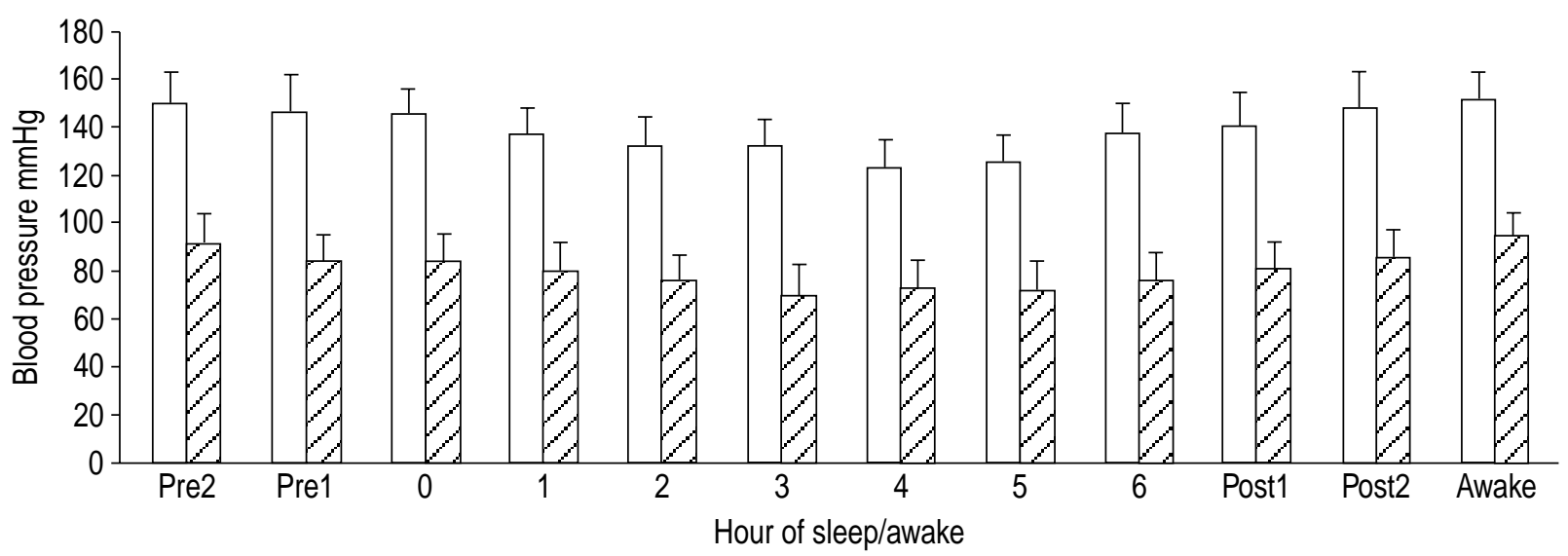

Fig. 2. - Hourly changes in systolic $(\square)$ and diastolic $(\square)$ blood pressures in pre-eclamptic subjects. Data are mean \pm SD. Pre1 and Pre2: values before sleep; Post1 and Post2: values after waking; Awake: values representing the mean of 4-hourly readings taken during the day while in hospital. Changes between awake and sleep are significant $(\mathrm{p}=0.001)$ for both systolic and diastolic pressures. 
Table 3. - Individual results in patients with pre-eclampsia

\begin{tabular}{|c|c|c|c|c|c|c|c|c|c|c|c|c|c|c|c|c|}
\hline \multirow[t]{2}{*}{ Variable } & \multicolumn{15}{|c|}{ Patient number } & \multirow[t]{2}{*}{ Mean \pm SD } \\
\hline & 1 & 2 & 3 & 4 & 5 & 6 & 7 & 8 & 9 & 10 & 11 & 12 & 13 & 14 & 15 & \\
\hline Age $y$ & 26 & 34 & 19 & 25 & 25 & 25 & 31 & 29 & 33 & 25 & 29 & 23 & 30 & 31 & 36 & $28 \pm 2.3$ \\
\hline nal & 271 & 209 & 264 & 280 & 186 & 292 & 266 & 203 & 263 & 292 & 280 & 276 & 222 & 263 & 259 & $255 \pm 33$ \\
\hline $\mathrm{BMI} \mathrm{kg} \cdot \mathrm{m}^{-2}$ & 34 & 28 & 32 & 34 & 30 & 33 & 32 & 33 & 32 & 33 & 32 & 30 & 29 & 24 & 30 & $31 \pm 1.3$ \\
\hline IFL \%SPT & 33 & 47 & 11 & 63 & 50 & 7 & 14 & 18 & 22 & 38 & 10 & 27 & 41 & 38 & 48 & $31 \pm 8.4$ \\
\hline IFL event duration min & 1.16 & 1.96 & 0.53 & 1.69 & 4.26 & 0.8 & 1.29 & 1.32 & 3.16 & 2.24 & 0.74 & 1.67 & 2.04 & 2.52 & 4.34 & $1.98 \pm 1.17$ \\
\hline Awake BP systolic $\mathrm{mmHg}$ & 149 & 157 & 145 & 160 & 159 & 143 & 156 & 151 & 148 & 161 & 156 & 152 & 153 & 144 & 150 & $152 \pm 5.8$ \\
\hline Aw: & 98 & 91 & 93 & 95 & 90 & 96 & 98 & 99 & 91 & 98 & 96 & 92 & 90 & 93 & 97 & $96 \pm 3.2$ \\
\hline Proteinuria $\mathrm{g} \cdot 24 \mathrm{~h}^{-1}$ & 0.4 & 0.4 & 0.35 & 0.4 & 1.2 & 1.0 & 0.5 & 0.45 & 0.6 & 0.75 & 1.0 & 0.85 & 0.35 & 0.3 & 0.5 & $0.6 \pm 0.3$ \\
\hline $\begin{array}{c}\text { Antihypertensive Rx } \\
\text { labetolol } 200 \mathrm{mg}\end{array}$ & $\mathrm{Y}$ & $\mathrm{N}$ & $\mathrm{Y}$ & $\mathrm{N}$ & $\mathrm{N}$ & $\mathrm{Y}$ & $\mathrm{N}$ & Y & $\mathrm{Y}$ & $\mathrm{N}$ & $\mathrm{N}$ & $\mathrm{Y}$ & $\mathrm{Y}$ & $\mathrm{Y}$ & $\mathrm{Y}$ & \\
\hline
\end{tabular}

BMI: body mass index; IFL: inspiratory flow limitation; \%SPT: per cent sleep period time; BP: blood pressure; Rx: therapy; N: no; Y: yes.

pre-eclampsia predates clinical manifestations of the disorder [4], which has been proposed as supportive evidence that snoring contributes to the development of pre-eclampsia. Support for a causative association comes from the recent report of EDWARDS et al. [10] who similarly demonstrated inspiratory flow limitation during sleep among a group of 11 patients with pre-eclampsia. These authors reported that abolition of inspiratory flow limitation by autosetting positive airway pressure over a single night resulted in significant reductions in BP during sleep.

The pathogenesis and mechanisms of pre-eclampsia are not well understood, but a generalized endothelial cell dysfunction is widely accepted to be a central feature [7-9]. Current concepts suggest that the pathogenesis relates to an imbalance of increased oxidative stress and lipid peroxidation, coupled to a deficiency of antioxidant protection, and also linked to impairment in placental perfusion. Interestingly, a number of maternal factors have been reported to increase the likelihood of developing pre-eclampsia [9], including obesity, insulin resistance, hypertension, and elevated plasma homocysteine levels. Several of these factors are also associated with the development of OSAS [15].

None of the subjects, including those with preeclampsia, showed evidence in sleep studies of a clinically significant sleep apnoea syndrome. The respiratory abnormality detected during approximately one-third of the night in pre-eclamptic patients is an indicator of flow limitation in the upper airway, and it has been proposed as an indicator of partial upper airway obstruction [11, 16-18]. Episodic inspiratory flow limitation without apnoeas or hypopnoeas can be associated with a clinical syndrome similar to OSAS, referred to as the upper airway resistance syndrome (UARS) [19], which may also respond to nCPAP therapy. However, the pattern of respiratory abnormality in UARS is one of transient episodes of inspiratory flow limitation, often terminated by arousal. In contrast, most of the patients demonstrated prolonged episodes of inspiratory flow limitation lasting several minutes (table 3).

The pathophysiological basis of inspiratory flow limitation cannot be determined precisely from the present study. This type of breathing pattern has been reported to occur in slow wave sleep, which has been reported to be increased in patients with pre-eclampsia [20] and can also be seen in the presence of loud snoring. Mouth-breathing can sometimes produce a breathing pattern on the Autoset device that could be mistaken for inspiratory flattening, but this can usually be distinguished from true inspiratory flow limitation by visual inspection of the original tracing, and was not the case in the present study.

Sleep staging was not performed during the sleep studies, and, therefore, the authors cannot comment on the stages of sleep in which inspiratory flow limitation occurred, nor can the authors be confident that patients slept for all of the sleep period time. Efforts were made to minimize the latter problem by asking patients to indicate with a buzzer, periods when they were consciously awake, which at least allowed the authors to exclude periods of obvious wakefulness. Furthermore, all subjects were observed by one of the investigators throughout the night. The patients with pre-eclampsia in the present study showed a fall in both systolic and diastolic BP levels during sleep, which is in contrast to some other reports that have demonstrated a rise, particularly in patients with severe pre-eclampsia [21, 22]. It is very unlikely that this difference could have biased the presented findings towards an increased incidence of sleep-related breathing disturbance in the pre-eclamptic group. The normal circadian pattern of BP variability is of a fall during sleep, whereas several reports have demonstrated a rise in BP during sleep in patients with sleep apnoea syndrome [6,23]. Thus, it could be argued that a pre-eclamptic group of patients that showed a rise in BP during sleep might be even more likely to be associated with evidence of inspiratory flow limitation during sleep. The degree of blunting of the normal circadian variation in BP among patients with pre-eclampsia appears to be related to the severity of the disorder [24], and those patients with an elevated nocturnal BP are likely to be the most severe.

The authors conclude that inspiratory flow limitation is common during sleep in females with 
pre-eclampsia in contrast to normal pregnant females. These findings may have implications for the pathophysiology and treatment of this disorder.

\section{References}

1. Young T, Palta M, Dempsey J, Skatrud J, Weber S, Badr S. The occurrence of sleep disordered breathing in middle-aged adults. N Engl J Med 1993; 328: 12301235.

2. Davies RJ, Stradling JR. The epidemiology of sleep apnoea. Thorax 1996; 51: Suppl. 2, S65-S70.

3. Loube MDI, Poceta JS, Morales MC, Peacock MD, Littner MM. Self-reported snoring in pregnancy: association with fetal outcome. Chest 1996; 109: 885-889.

4. Franklin KA, Holmgren PA, Jonsson F, Poromaa N, Stenlund H, Svanborg E. Snoring, pregnancy-induced hypertension, and growth retardation of the fetus. Chest 2000; 117: 137-141.

5. Young T, Peppard P, Palta M, et al. Population-based study of sleep disordered breathing as a risk factor for hypertension. Arch Intern Med 1997; 157: 1746-1752.

6. Fletcher EC. The relationship between systemic hypertension and obstructive sleep apnea: facts and theory. Am J Med 1995; 98: 118-128.

7. Broughton Pipkin F. The hypertensive disorders of pregnancy. BMJ 1995; 311: 609-613.

8. Perloff D. Hypertension and pregnancy related hypertension. Cardiol Clin 1998; 16: 79-101.

9. Walsh SW. Maternal-placental interactions of oxidative stress and antioxidants in preeclampsia. Semin Reprod Endocrin 1998; 16: 93-104.

10. Edwards N, Blyton DM, Kirjavainen T, Kesby GJ, Sullivan CE. Nasal continuous positive airway pressure reduces sleep-induced blood pressure increments in preeclampsia. Am J Respir Crit Care Med 2000; 162: 252-257.

11. Teschler H, Berthon-Jones M, Thompson A, Henkel A, Henry J, Konietzko N. Automated continuous positive airway pressure titration for obstructive sleep apnea syndrome. Am J Respir Crit Care Med 1996; 154: 734-740.

12. Gugger M, Mathis $\mathrm{J}$, Bassetti C. Accuracy of an intelligent CPAP machine with in-built diagnostic abilities in detecting apnoeas: a comparison with polysomnography. Thorax 1995; 50: 1199-1201.
13. Kiely JL, Delahunty C, Matthews S, McNicholas WT. Comparison of a limited computerized diagnostic system (Rescare Autoset) with polysomnography in the diagnosis of obstructive sleep apnoea syndrome. Eur Respir J 1996; 9: 2360-2364.

14. Davies RJ, Jenkins NE, Stradling JR. Effect of measuring ambulatory blood pressure on sleep and on blood pressure during sleep. BMJ 1994; 308: 820823.

15. Rosenow F, McCarthy V, Caruso AC. Sleep apnoea in endocrine diseases. J Sleep Res 1998; 7: 3-11.

16. Hosselet JJ, Norman RG, Ayappa I, Rapoport DM. Detection of flow limitation with a nasal cannula/ pressure transducer system. Am J Respir Crit Care Med 1998; 157: 1461-1467.

17. Montserrat JM, Ballester E, O1ivi H, et al. Timecourse of stepwise CPAP titration. Am J Respir Crit Care Med 1995; 152: 1854-1859.

18. The Report of a Task Force of the American Academy of Sleep Medicine. Sleep-related breathing disorders in adults: recommendations for syndrome definition and measurement techniques in clinical research. Sleep 1999; 22: 667-689.

19. Guilleminault C, Stoohs R, Clerk A, Cetel M, Maistros P. A cause of excessive daytime sleepiness: the upper airway resistance syndrome. Chest 1993; 104: 781-787.

20. Edwards N, Blyton DM, Kesby GJ, Wilcox I, Sullivan CE. Pre-eclampsia is associated with marked alterations in sleep architecture. Sleep 2000; 23: 619-625.

21. Sawyer MM, Lipshitz J, Anderson GD, Dilts PV Jr, Halperin L. Diurnal and short-term variation of blood pressure: comparison of preeclamptic, chronic hypertensive, and normotensive patients. Obstet Gynecol 1981; 58: 291-296.

22. Oney T, Meyer-Sabellek W. Variability of arterial blood pressure in normal and hypertensive pregnancy. J Hypertens Suppl 1990; 8: S77-S81.

23. Davies RJO, Crosby J, Prothero A, Stradling JR. Ambulatory blood pressure and left ventricular hypertrophy in untreated obstructive sleep apnoea and snoring, compared to matched controls, and their response to treatment. Clin Sci 1994; 86: 417-424.

24. Halligan A, Shennan A, Lambert PC, de Swiet M, Taylor DJ. Diurnal blood pressure difference in the assessment of preeclampsia. Obstet Gynecol 1996; 87: 205-208. 